\title{
Quantitative Chest CT analysis in discriminating COVID-19 from non-COVID-19 patients
}

\author{
Damiano Caruso ${ }^{1} \cdot$ Michela Polici $^{1} \cdot$ Marta Zerunian $^{1} \cdot$ Francesco Pucciarelli $^{1} \cdot$ Tiziano Polidori $^{1} \cdot$ Gisella Guido $^{1}$. \\ Carlotta Rucci ${ }^{1}$. Benedetta Bracci ${ }^{1} \cdot$ Emanuele Muscogiuri $^{1} \cdot$ Chiara De Dominicis $^{1} \cdot$ Andrea Laghi $^{1}$ (1)
}

Received: 8 July 2020 / Accepted: 13 September 2020 / Published online: 12 October 2020

(c) Italian Society of Medical Radiology 2020

\begin{abstract}
Introduction COVID-19 pneumonia is characterized by ground-glass opacities (GGOs) and consolidations on Chest CT, although these CT features cannot be considered specific, at least on a qualitative analysis. The aim is to evaluate if Quantitative Chest CT could provide reliable information in discriminating COVID-19 from non-COVID-19 patients.

Materials and methods From March 31, 2020 until April 18, 2020, patients with Chest CT suggestive for interstitial pneumonia were retrospectively enrolled and divided into two groups based on positive/negative COVID-19 RT-PCR results. Patients with pulmonary resection and/or CT motion artifacts were excluded. Quantitative Chest CT analysis was performed with a dedicated software that provides total lung volume, healthy parenchyma, GGOs, consolidations and fibrotic alterations, expressed both in liters and percentage. Two radiologists in consensus revised software analysis and adjusted areas of lung impairment in case of non-adequate segmentation. Data obtained were compared between COVID-19 and non-COVID-19 patients and $p<0.05$ were considered statistically significant. Performance of statistically significant parameters was tested by ROC curve analysis.

Results Final population enrolled included 190 patients: 136 COVID-19 patients (87 male, 49 female, mean age 66 \pm 16 ) and 54 non-COVID-19 patients ( 25 male, 29 female, mean age $63 \pm 15$ ). Lung quantification in liters showed significant differences between COVID-19 and non-COVID-19 patients for GGOs $(0.55 \pm 0.26 \mathrm{~L}$ vs $0.43 \pm 0.23 \mathrm{~L}, p=0.0005)$ and fibrotic alterations $(0.05 \pm 0.03 \mathrm{~L}$ vs $0.04 \pm 0.03 \mathrm{~L}, p<0.0001)$. ROC analysis of GGOs and fibrotic alterations showed an area under the curve of 0.661 (cutoff $0.39 \mathrm{~L}, 68 \%$ sensitivity and $59 \%$ specificity, $p<0.001$ ) and 0.698 (cutoff $0.02 \mathrm{~L}, 86 \%$ sensitivity and $44 \%$ specificity, $p<0.001)$, respectively.
\end{abstract}

Conclusions Quantification of GGOs and fibrotic alterations on Chest CT could be able to identify patients with COVID-19.

Keywords COVID-19 $\cdot$ Chest CT $\cdot$ Quantitative Chest CT analysis $\cdot$ Interstitial pneumonia

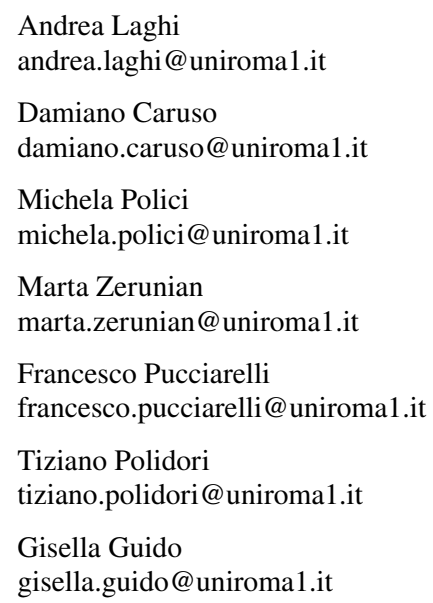

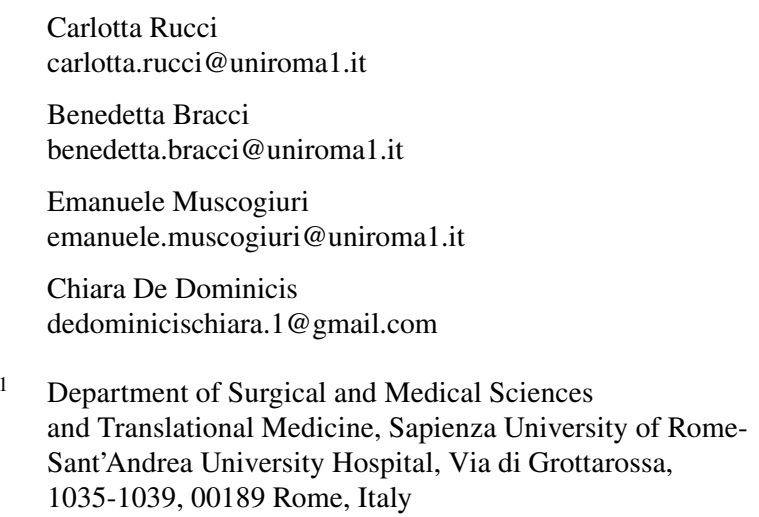

Department of Surgical and Medical Sciences and Translational Medicine, Sapienza University of RomeSant'Andrea University Hospital, Via di Grottarossa, 1035-1039, 00189 Rome, Italy 


\begin{tabular}{|c|c|}
\hline \multicolumn{2}{|c|}{ Abbreviations } \\
\hline SARS-CoV-2 & $\begin{array}{l}\text { Severe Acute Respiratory Syndrome } \\
\text { Coronavirus } 2\end{array}$ \\
\hline COVID-19 & Coronavirus Disease 2019 \\
\hline RT-PCR & $\begin{array}{l}\text { Reverse transcription polymerase chain } \\
\text { reaction }\end{array}$ \\
\hline GGOs & Ground-glass opacities \\
\hline CRP & C-reactive protein \\
\hline LDH & Lactate dehydrogenase \\
\hline
\end{tabular}

\section{Introduction}

From the last months of 2019 the novel coronavirus, severe acute respiratory syndrome Coronavirus 2 (SARS-CoV-2) [1], has led a pandemic diffusion all over the World [2] and the disease associated was named Coronavirus Disease 2019 (COVID-19) [3]. Guidelines recommend an upper respiratory specimen that should be tested with next-generation sequencing or real-time reverse transcription polymerase chain reaction (RT-PCR) methods for diagnosis of COVID19 [4], but the early diagnosis is affected by low sensitivity $(60-71 \%)[5,6]$ of the test, due to errors in laboratory work or sample collection [7]; moreover, RT-PCR is not universally available, especially in countries with limited resources.

CT was demonstrated to have a pivotal role in the COVID-19 pandemic thanks to a sensitivity of $97 \%$ [8], and might be helpful in the management [9] and follow-up of COVID-19 patients [10]; however, due to the low specificity, false positive patients were encountered in clinical practice leaving CT unreliable for the diagnosis [11, 12]. A recent consensus statement from the Fleischner Society pointed out as imaging is indicated for medical triage of suspected COVID-19 patients presenting moderate-severe clinical features and a high pre-test probability of disease [13].

Recently, severity lung scores $[14,15]$ were proposed from a visual and qualitative evaluation of CT features, but several limitations were reported (i.e., subjectivity, lack of standardization and reproducibility) based on radiologist's experience [16, 17].

Quantitative Chest CT analysis has the ability to provide quantitative and objective assessment of the healthy lungs, consolidations and ground-glass opacities (GGOs) necessary to quantify the disease burden $[17,18]$. In addition, a quantitative analysis could provide relevant information on the progression of the disease and the response to therapy in the follow-up examinations.

During the pandemic, GGOs and consolidations were frequently observed; nevertheless, these CT features were not specific for COVID-19. Thus, the aim of our study was to evaluate if Quantitative Chest CT could provide reliable information in discriminating RT-PCR COVID-19 from non-COVID-19 patients.

\section{Materials and methods}

\section{Patient population}

This retrospective study was approved by our local institutional review board (IRB) and written informed consent was obtained from all patients or in case of inability from their relatives or the admitting physicians. Patients admitted at the Emergency Department of Sant'Andrea Hospital in Rome from March 31, 2020 until April 18, 2020 were retrospectively included in the study.

Inclusion criteria were (a) diagnosis of interstitial pneumonia on Chest CT and (b) availability of RT-PCR results for COVID-19. Patients with history of lung malignancy who required pulmonary resection and oncohaematologic patients were excluded from the analysis. Image datasets affected by severe respiratory artifacts in whom the software was unable to process the data were also excluded.

Based on RT-PCR results, our population was divided in COVID-19 and non-COVID-19.

\section{Clinical data}

According to the hospital internal protocol, at the time of admission suspected COVID-19 patients presenting moderate-severe clinical features and a high pre-test probability of disease (fever defined as $>37.5{ }^{\circ} \mathrm{C}$ and respiratory symptoms or direct contact with a confirmed COVID-19 patient) underwent nasopharyngeal and oropharyngeal swabs for SARS-CoV-2. Every patient was tested with two nasopharyngeal and oropharyngeal swabs, the first swab at the entrance and the second after $24 \mathrm{~h}$. The positivity to SARS-CoV-2 was obtained with RT-PCR (Charitè, Berlin, Germany) [19], whereas patients were considered SARSCoV-2 negative after two consecutive negative RT-PCR results. Demographic data and laboratory results were also collected.

\section{CT acquisition technique}

After the first swab, all suspected COVID-19 patients underwent Chest CT to evaluate the presence of interstitial pneumonia. Chest CT was acquired without contrast medium and in supine position during end-inspiration. Each patient was studied using a COVID-19 dedicated 128-slice CT (GE Revolution EVO 64 Slice CT Scanner, GE Medical Systems, Milwaukee, WI, USA). CT scan technical parameters: tube voltage: $120 \mathrm{kV}$; tube current modulation: 100-250 mAs; spiral pitch factor: 0.98; collimation width: 0.625 . 
Reconstruction images were performed with convolution kernel BONEPLUS at a slice thickness of $1.25 \mathrm{~mm}$. After completion of the CT examination the decontamination of the room was made for every patient, it was performed throughout passive air exchange and disinfecting the surfaces with $0.1 \%$ sodium hypochlorite or $62-71 \%$ ethanol.

\section{Quantitative Chest CT analysis}

Two radiologists in consensus (MZ and FP with 5 and 4 years of experience), blinded to clinical data and RT-PCR results, performed Quantitative Chest $\mathrm{CT}$ analysis by using a dedicated software (Thoracic VCAR v13.1, GE). Before segmentation, attenuation value $<-1000 \mathrm{HU}$ was used to exclude trachea air from the analysis. Quantification was performed on naive acquisition using a lung window with a width of $1500 \mathrm{HU}$ and a level of $-600 \mathrm{HU}$. The software automatically calculated the following parameters: healthy parenchyma, GGOs, consolidation, fibrotic alterations (including fibrotic stripes and subpleural lines) using an adaptive mean based on grayscale, expressed both in liters and percentages, whereas the total lung volume was reported in liters. Vessel was automatically selected and delated. In case of non-adequate automatic segmentation, readers were free to adjust the area of lung impairment segmented by the software.

\section{Statistical analysis}

Statistical analysis was performed using MedCalc Statistical Software version 17.9.7 (MedCalc Software bvba, Ostend, Belgium), and $p$ values $<0.05$ were considered statistically significant. All data are expressed as mean \pm standard deviation (SD). To compare the two group of COVID-19 and nonCOVID-19 patients, Kolmogorov-Smirnov test was used to assess data distribution. In case of Gaussian distribution, data were tested with Student's $t$ test, while Wilcoxon test was applied for non-Gaussian distributed data. Receiver operating characteristic (ROC) curves and Area under the curve (AUC) were calculated for predicting the performance of the quantitative analysis. Results are provided both in liters and percentages.

\section{Results}

\section{Patient population and clinical data}

Two-hundred and sixteen patients met the inclusion criteria, whereas 26 patients were excluded because of image artefacts. Thus, our final population comprised 190 patients: 136 COVID- 19 patients ( 87 males and 49 females, mean age $66 \pm 16$ years) and 54 non-COVID-19 patients ( 25 males and 29 females, mean age $63 \pm 15$ years) as shown in Fig. 1 .

Considering COVID-19 patients, 80/136 patients (59\%) presented fever $\left(>37.5^{\circ} \mathrm{C}\right), 49 / 136(36 \%)$ cough, and 47/136 (34\%) dyspnea. Laboratory tests showed lymphocytopenia in $106 / 136$ patients $(78 \%)$; high values $(>0.50 \mathrm{mg} / \mathrm{dL})$ of C-reactive protein (CRP), lactate dehydrogenase (LDH) $(>220 \mathrm{U} / \mathrm{L})$ and $\mathrm{D}$-dimer $(>243 \mathrm{ng} / \mathrm{ml})$ were found in $128 / 136$ patients (94\%), $122 / 136$ patients $(90 \%)$ and $87 / 136$ patients, respectively.

Regarding non-COVID-19 patients, $26 / 54$ patients (48\%) had fever $\left(>37.5^{\circ} \mathrm{C}\right), 20 / 54(37 \%)$ cough and 13/54 (24\%) dyspnea. Lymphocytopenia was reported in $32 / 54$ patients (59\%); high values ( $>0.50 \mathrm{mg} / \mathrm{dL})$ of CRP, LDH $(>220$ $\mathrm{U} / \mathrm{L}$ ) and D-dimer ( $>243 \mathrm{ng} / \mathrm{ml}$ ) were found in $41 / 54$ patients (76\%), 40/54 patients (74\%) and 33/54 patients (61\%), respectively. Full results are listed in Table 1.

\section{Quantitative Chest CT analysis}

Considering results expressed in liters, significant differences were reported for GGOs $(0.55 \pm 0.26 \mathrm{~L}$ vs $0.43 \pm 0.23$ $\mathrm{L}, p=0.0005)$ and fibrotic alterations $(0.05 \pm 0.03 \mathrm{~L}$ vs $0.04 \pm 0.03 \mathrm{~L}, p<0.0001$ ) (Fig. 2). No significant differences were found for total lung volume $(4.09 \pm 1.49 \mathrm{~L}$ vs $4.20 \pm 1.28 \mathrm{~L}, p=0.37)$, healthy parenchyma $(3.36 \pm 1.58 \mathrm{~L}$ vs $3.64 \pm 1.33 \mathrm{~L}, p=0.11)$ and consolidations $(0.06 \pm 0.04 \mathrm{~L}$ vs $0.06 \pm 0.03 \mathrm{~L}, p=0.31$ ). No significant differences were

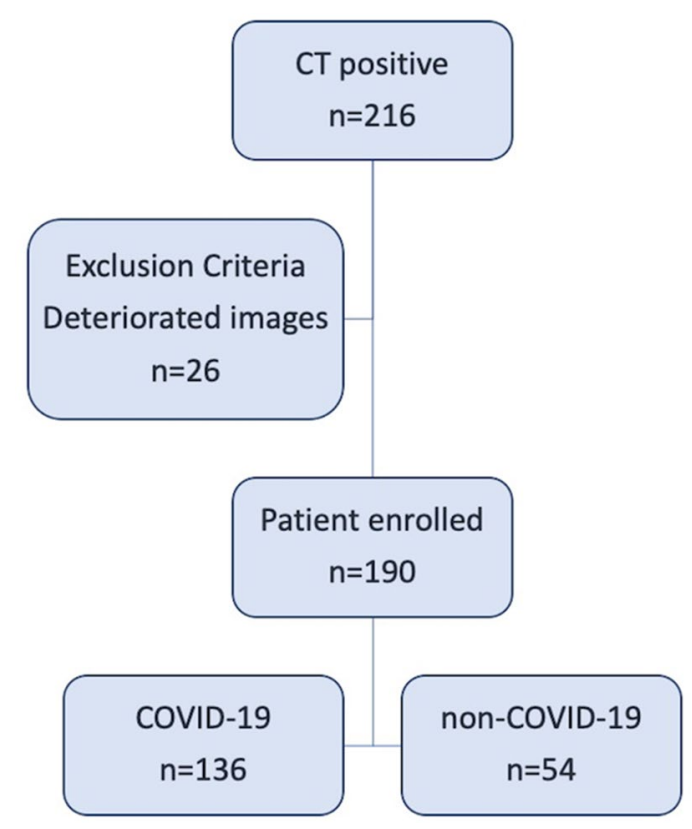

Fig. 1 Flowchart of the study. From the initial population of 216 Chest CT positive for interstitial pneumonia, we enrolled 136 patients COVID-19 and 54 patients non-COVID-19 
Table 1 Clinical data

Fig. 2 57-year-old man with COVID-19 (a, b) and 58-yearold man non-COVID-19 (c, d). Axial unenhanced quantified Chest CT scans that show diffuse bilateral groundglass opacities (GGOs) and some fibrotic alterations in COVID-19 patient (a, b) and rare ground-glass opacities in non-COVID-19 patient (c, d). Chest CT semi-automatic quantification shows GGOs in red, vessels in yellow and fibrotic alterations in blue, these findings are more represented in COVID-19 patient (a) then in non-COVID-19 patient (c)

\begin{tabular}{|c|c|c|c|c|}
\hline & \multicolumn{2}{|l|}{ COVID-19 } & \multicolumn{2}{|c|}{ Non-COVID-19 } \\
\hline & N. patients & $\%$ & N. patients & $\%$ \\
\hline \multicolumn{5}{|c|}{ Patients demographics } \\
\hline Mean age & $66 \pm 16$ year & & $63 \pm 15$ year & \\
\hline Years (range) & $18-97$ & & $18-96$ & \\
\hline Number patients & 136 & 100 & 54 & 100 \\
\hline Male & $87 / 136$ & 64 & $25 / 54$ & 46 \\
\hline Female & $49 / 136$ & 36 & $29 / 54$ & 54 \\
\hline \multicolumn{5}{|l|}{ Blood test } \\
\hline \multicolumn{5}{|c|}{ C-reactive protein $(\mathrm{mg} / \mathrm{L}$; normal range $0.00-0.50)$} \\
\hline Increased & $128 / 136$ & 94 & $41 / 54$ & 76 \\
\hline Normal & $8 / 136$ & 7 & $13 / 54$ & 24 \\
\hline \multicolumn{5}{|c|}{ Lactic Acid Dehydrogenase (U/L; range 125-220) } \\
\hline Increased & $122 / 136$ & 90 & $40 / 54$ & 74 \\
\hline Normal & $14 / 136$ & 10 & $14 / 54$ & 26 \\
\hline \multicolumn{5}{|c|}{ Lymphocytes $\left(\times 10^{3} / \mathrm{mm}^{3}\right.$, normal range $\left.1.5-3.0\right)$} \\
\hline Increased & $1 / 136$ & 0.7 & $4 / 54$ & 7 \\
\hline Decreased & $106 / 136$ & 78 & $32 / 54$ & 59 \\
\hline Normal & $29 / 136$ & 21.3 & $18 / 54$ & 34 \\
\hline \multicolumn{5}{|c|}{ D-dimer $(\mathrm{ng} / \mathrm{ml}$, normal <243) } \\
\hline Increased & $87 / 136$ & 64 & $33 / 54$ & 61 \\
\hline Normal & $49 / 136$ & 36 & $21 / 54$ & 39 \\
\hline \multicolumn{5}{|l|}{ Symptoms } \\
\hline Fever $\left(>38^{\circ}\right)$ & $80 / 136$ & 59 & $26 / 54$ & 48 \\
\hline Cough & $49 / 136$ & 36 & $20 / 54$ & 37 \\
\hline Dyspnea & $47 / 136$ & 34 & $13 / 54$ & 24 \\
\hline
\end{tabular}

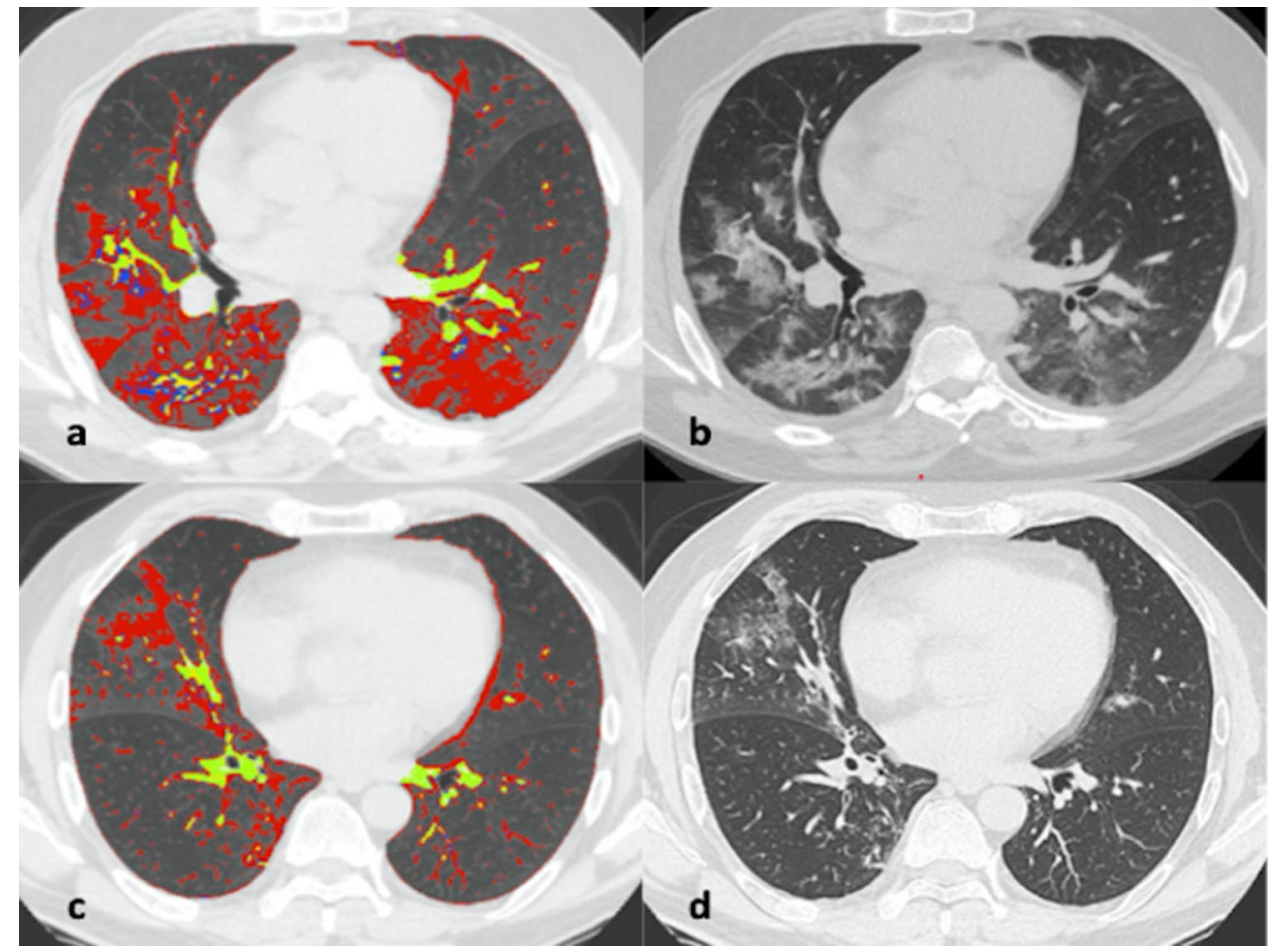


found in the evaluations of lung parenchyma expressed in percentage. Full results are shown in Table 2.

ROC analysis performed for GGOs and fibrotic alterations expressed in liters returned an area under the curve (AUC) of 0.661 for GGOs and 0.698 for fibrotic alterations. When the cutoff of GGOs was set at 0.39L ( $p<0.001)$, sensitivity and specificity were $68 \%$ and $59 \%$, respectively, while sensitivity and specificity of fibrotic alterations were $86 \%$ and $44 \%$, respectively with a cutoff of $0.02 \mathrm{~L}(p<0.001)$ (Fig. 3).

\section{Discussion}

The results of our study showed a good performance of semi-automatic lung quantification in distinguishing symptomatic patients affected by COVID-19 from those with interstitial pneumonia from non-COVID-19 causes. In particular, quantification of GGOs and fibrotic alterations (including fibrotic stripes and subpleural lines), although with moderate sensitivity and specificity, may be helpful, whereas healthy parenchyma and consolidations are not.

Our results disagree with some literature data, where GGOs are described as the most frequent initial findings in COVID-19 pneumonia [8, 20], but they are not considered specific for COVID-19 [8]. In fact, Chen D. and colleagues analyzed both RT-PCR positive and negative patients in a preliminary study involving 21 patients and their results showed presence of GGOs for 20/21 (95\%) patients, with no differences between positive and negative RT-PCR results ( $p=0.469)$, while significant lower presence of consolidation ( $p=0.04)$ was observed in negative RT-PCR patients [21]. The discrepancies between our results and literature data might be explained by several factors: firstly, during early disease spread RT-PCR tests were even less sensitive than now, and the possibility of false negative in a population enrolled during January and February 2020 was concrete with consequent bias on Chest CT findings [22]; then reduced sample size analyzed by authors could have influenced the statistical prevalence of results obtained. In addition, the different imaging analysis, visual or qualitative
Table 2 Quantitative Chest CT analysis
Fig. 3 ROC curves to test the ability of ground-glass opacities and fibrotic alterations in differentiating COVID-19 from non-COVID-19 patients

\begin{tabular}{lccc}
\hline & $\begin{array}{l}\text { COVID-19 } \\
\text { Mean }\end{array}$ & $\begin{array}{l}\text { Non-COVID-19 } \\
\text { Mean }\end{array}$ & $P$ values \\
\hline Quantitative analysis in liters & & & \\
$\quad$ Total lung volume & $4.09 \pm 1.49$ & $4.20 \pm 1.28$ & 0.37 \\
Healthy parenchyma & $3.36 \pm 1.58$ & $3.64 \pm 1.33$ & 0.11 \\
Ground-glass opacities & $0.55 \pm 0.26$ & $0.43 \pm 0.23$ & $\mathbf{0 . 0 0 0 5}$ \\
Fibrotic alterations & $0.05 \pm 0.03$ & $0.04 \pm 0.03$ & $<\mathbf{0 . 0 0 0 1}$ \\
Consolidations & $0.06 \pm 0.04$ & $0.06 \pm 0.03$ & 0.31 \\
Quantitative analysis in percentage & & & 0.36 \\
Healthy parenchyma & $79.35 \pm 13.54$ & $85.09 \pm 9.35$ & 0.47 \\
Ground-glass opacities & $15.59 \pm 9.65$ & $11.54 \pm 7.47$ & 0.06 \\
Fibrotic alterations & $1.40 \pm 1.07$ & $0.93 \pm 0.77$ & 0.91 \\
Consolidations & $1.75 \pm 1.47$ & $1.53 \pm 1.03$ & \\
\hline
\end{tabular}

Significant differences are shown in bold

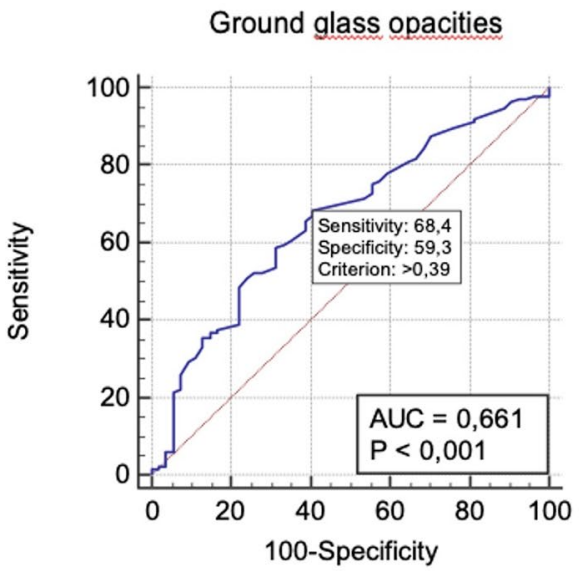


versus quantitative assessment could be explain the differences. Qualitative analysis is also affected by radiologist skill and experience in image interpretation, whereas quantitative evaluation is a reproducible and comparable technique.

Our results, however, differ also from published studies using a quantitative approach. In a recent paper, a combined scoring system (clinical and radiological features) based on visual assessment was proposed to differentiate COVID-19 patients from interstitial pneumonia of other etiology [23]. Authors interestingly concluded that the combined CT features analysis revealed an AUC of 0.854. Our single-parameter showed an AUC of 0.661 for GGOs and of 0.698 for fibrotic alterations. Despite GGOs performance did not reach the AUC of the cited combined model, this single quantified parameter indicated that it could be a good parameter for the identification of COVID-19 patients just with the sole $\mathrm{CT}$ imaging technique. This might be useful in an resourceconstrained setting when rapid point-of-care COVID-19 testing is not available or negative. As stated by Fleischner Society, in high pre-test probability scenarios, presumptive diagnosis of COVID-19 achieved by imaging features might support medical triage and clinical management [22]. Moreover, despite these indications are not exactly in line with international guidelines which consider RT-PCR as reference standard for diagnosis, direct experience in critical scenarios revealed how Chest CT represents an helpful resource to suspect a COVID-19 case before the RT-PCR tests; in fact, the latter require an unsustainable response time in a scenario where urgent decision-making is a priority with high number of new hospital admissions associated with limited health personnel and infrastructure resources.

Several controversies emerged on interpretation and frequency of fibrotic alterations. Some authors have described fibrotic alterations as atypical findings, observed on Chest CT only in $1.6 \%$ of the population studied [24]; on the contrary, Lomoro et al. [25] considered fibrotic alterations as a typical CT findings due to their presence in $50 \%$ of COVID-19 patients at hospital admission. In accordance with Lomoro P. and colleagues, our results showed higher percentage of fibrotic alterations in COVID-19 patients than non-COVID-19 $(p<0.0001)$. Discrepancies among studies might have different explanations: first of all, different CT pulmonary evaluations (visual vs quantitative) could have affect how CT features were assessed; then, a possible bias could be the time range between symptoms onset and hospital admission when CT scans were performed. In fact, the longer the time between the onset of symptoms and the acquisition of CT scan, the higher the probability to detect fibrotic alterations, indicative of a late stage pneumonia.

Despite the interesting results, the present study has some limitations such as the retrospective nature, lack of data regarding symptoms onset from the hospital admission, the small sample of patients with negative RT-PCR included and the lack of a combined clinical and radiological decisional model to discriminate COVID-19 from non-COVID-19 patients.

\section{Conclusions}

In conclusion, Chest $\mathrm{CT}$ quantification has the potential to improve COVID-19 management for diagnosis; it could increase specificity of CT images and help the identification of COVID-19 patients before the RT-PCR results. By so doing, it could be a very useful tool in difficult scenarios such as resource-constrained setting triage. Future application might also include COVID-19 CT follow-up with a quantitative assessment of lung impairment as expression of worsening, stability or healing of lung parenchyma. To explore these possibilities, larger studies and data interpretation are needed.

CT quantification of ground-glass opacities and fibrotic alterations is able to identify COVID-19 patients with moderate accuracy and may help radiologists to overcome low specificity of Chest CT.

Acknowledgements We would like also to acknowledge the entire Radiological Medical and Technical Staff of the Radiology Unit of Sant'Andrea Academic Hospital in Rome.

Authors' contribution D. Caruso: Project administration, Formal analysis, Writing — review \& editing; M. Polici: Data curation, Writingoriginal draft; M. Zerunian: Methodology, Writing—original draft; F. Pucciarelli: Software; T. Polidori: Visualization; G. Guido: Validation; C. Rucci: Software; B. Bracci: Methodology; E. Muscogiuri: Visualization; C. De Dominicis: Conceptualization; A. Laghi: Supervision, Formal analysis, Writing - review \& editing.

Funding None.

Availability of data and materials The datasets during and/or analyzed during the current study available from the corresponding author on reasonable request.

\section{Compliance with ethical standards}

Conflict of interest The authors declare that they have no conflict of interest.

Consent to participate Informed consent was obtained from all individual participants included in the study

Consent for publication The authors affirm that human research participants provided informed consent for publication of the images in Fig. 2.

Code availability Quantitative Chest CT analysis was performed by using a dedicated software (Thoracic VCAR v13.1, GE). 
Ethics approval This retrospective study was approved by our local institutional review board (IRB) and written informed consent was obtained from all patients or in case of inability from their relatives or the admitting physicians.

\section{References}

1. Naming the coronavirus disease (COVID-19) and the virus that causes it (2020). https://www.who.int/emergencies/diseases/novel -coronavirus-2019/technical-guidance/naming-the-coronaviru s-disease-(covid-2019)-and-the-virus-that-causes-it

2. Coronavirus disease (COVID-2019) situation reports (2020). https ://www.who.int/emergencies/diseases/novel-coronavirus-2019/situa tion-reports

3. Novel Coronavirus - China (2020). https://www.who.int/csr/don/12january-2020-novel-coronavirus-china/en/

4. Interim Guidance: Healthcare Professionals 2019-nCoVICDC (2020). https://www.cdc.gov/coronavirus/2019-ncov/hcp/clinicalcriteria.html

5. Yang Yang MY, Shen Chenguang, Wang Fuxiang, Yuan Jing, Li Jinxiu, Zhang Mingxia, Wang Zhaoqin, Xing Li, Wei Jinli, Peng Ling, Wong Gary, Zheng Haixia, Weibo Wu, Liao Mingfeng, Feng Kai, Li Jianming, Yang Qianting, Zhao Juanjuan, Zhang Zheng, Liu Lei, Liu Yingxia (2020) Evaluating the accuracy of different respiratory specimens in the laboratory diagnosis and monitoring the viral shedding of 2019-nCoV infections. medRxiv 1:5. https:// doi.org/10.1101/2020.02.11.20021493

6. Kanne JP, Little BP, Chung JH, Elicker BM, Ketai LH (2020) Essentials for radiologists on COVID-19: an update. Radiology. https:// doi.org/10.1148/radiol.2020200527

7. Xie X, Zhong Z, Zhao W, Zheng C, Wang F, Liu J (2020) Chest CT for typical 2019-nCoV pneumonia: relationship to negative RT-PCR testing. Radiology. https://doi.org/10.1148/radiol.2020200343

8. Ai T, Yang Z, Hou H, Zhan C, Chen C, Lv W, Tao Q, Sun Z, Xia L (2020) Correlation of chest CT and RT-PCR testing in coronavirus disease 2019 (COVID-19) in China: a report of 1014 cases. Radiology. https://doi.org/10.1148/radiol.2020200642

9. Watanabe M, Caruso D, Tuccinardi D, Risi R, Zerunian M, Polici M, Pucciarelli F, Tarallo M, Strigari L, Manfrini S, Mariani S, Basciani S, Lubrano C, Laghi A, Gnessi L (2020) Visceral fat shows the strongest association with the need of intensive care in patients with COVID-19. Metabolism. https://doi.org/10.1016/j.metab ol.2020.154319

10. Li Y, Xia L (2020) Coronavirus disease 2019 (COVID-19): role of chest CT in diagnosis and management. AJR Am J Roentgenol 1:1-7. https://doi.org/10.2214/ajr.20.22954

11. Kim H, Hong H, Yoon SH (2020) Diagnostic performance of CT and reverse transcriptase-polymerase chain reaction for coronavirus disease 2019: a meta-analysis. Radiology. https://doi.org/10.1148/ radiol.2020201343

12. Caruso D, Zerunian M, Polici M, Pucciarelli F, Polidori T, Rucci C, Guido G, Bracci B, de Dominicis C, Laghi A (2020) Chest CT features of COVID-19 in Rome, Italy. Radiology. https://doi. org/10.1148/radiol.2020201237

13. Rubin GD, Ryerson CJ, Haramati LB, Sverzellati N, Kanne JP, Raoof S, Schluger NW, Volpi A, Yim JJ, Martin IBK, Anderson DJ, Kong C, Altes T, Bush A, Desai SR, Goldin J, Goo JM, Humbert M, Inoue Y, Kauczor HU, Luo F, Mazzone PJ, Prokop M, RemyJardin M, Richeldi L, Schaefer-Prokop CM, Tomiyama N, Wells AU, Leung AN (2020) The role of chest imaging in patient management during the COVID-19 pandemic: a multinational consensus statement from the Fleischner Society. Radiology. https://doi. org/10.1148/radiol.2020201365

14. Bai HX, Hsieh B, Xiong Z, Halsey K, Choi JW, Tran TML, Pan I, Shi LB, Wang DC, Mei J, Jiang XL, Zeng QH, Egglin TK, Hu PF, Agarwal S, Xie F, Li S, Healey T, Atalay MK, Liao WH (2020)
Performance of radiologists in differentiating COVID-19 from viral pneumonia on chest CT. Radiology. https://doi.org/10.1148/radio 1.2020200823

15. Huang G, Gong T, Wang G, Wang J, Guo X, Cai E, Li S, Li X, Yu Y, Lin L (2020) Timely diagnosis and treatment shortens the time to resolution of coronavirus disease (COVID-19) pneumonia and lowers the highest and last CT scores from sequential chest CT. AJR Am J Roentgenol. https://doi.org/10.2214/ajr.20.23078

16. Shen C, Yu N, Cai S, Zhou J, Sheng J, Liu K, Zhou H, Guo Y, Niu G (2020) Quantitative computed tomography analysis for stratifying the severity of coronavirus disease 2019. J Pharm Anal. https://doi. org/10.1016/j.jpha.2020.03.004

17. Lu Huang RH, Ai Tao, Pengxin Yu, Kang Han, Tao Qian, Xia Liming (2020) Serial quantitative chest CT assessment of COVID19: deep-learning approach. Radiology. https://doi.org/10.1148/ ryct.2020200075

18. Ran Yang XL, Liu Huan, Zhen Yanling, Zhang Xianxiang, Xiong Qiuxia, Luo Yong, Gao Cailiang, Zeng Wenbing (2020) Chest CT severity score: an imaging tool for assessing severe COVID-19. Radiology. https://doi.org/10.1148/ryct.2020200047

19. Corman VM, Landt O, Kaiser M, Molenkamp R, Meijer A, Chu DK, Bleicker T, Brünink S, Schneider J, Schmidt ML, Mulders DG, Haagmans BL, van der Veer B, van den Brink S, Wijsman L, Goderski G, Romette JL, Ellis J, Zambon M, Peiris M, Goossens H, Reusken C, Koopmans MP, Drosten C (2020) Detection of 2019 novel coronavirus (2019-nCoV) by real-time RT-PCR. Euro Surveill 25(3):2000045. https://doi.org/10.2807/1560-7917. es.2020.25.3.2000045

20. Chung M, Bernheim A, Mei X, Zhang N, Huang M, Zeng X, Cui J, Xu W, Yang Y, Fayad ZA, Jacobi A, Li K, Li S, Shan H (2020) CT imaging features of 2019 novel coronavirus (2019-nCoV). Radiology 295(1):202-207. https://doi.org/10.1148/radiol.2020200230

21. Chen D, Jiang X, Hong Y, Wen Z, Wei S, Peng G, Wei X (2020) Can chest $\mathrm{CT}$ features distinguish patients with negative from those with positive initial RT-PCR results for coronavirus disease (COVID19)? AJR Am J Roentgenol. https://doi.org/10.2214/ajr.20.23012

22. Rubin GD, Ryerson CJ, Haramati LB, Sverzellati N, Kanne JP, Raoof S, Schluger NW, Volpi A, Yim JJ, Martin IBK, Anderson DJ, Kong C, Altes T, Bush A, Desai SR, Goldin J, Goo JM, Humbert M, Inoue Y, Kauczor HU, Luo F, Mazzone PJ, Prokop M, Remy-Jardin M, Richeldi L, Schaefer-Prokop CM, Tomiyama N, Wells AU, Leung AN (2020) The role of chest imaging in patient management during the COVID-19 pandemic: a multinational consensus statement from the Fleischner Society. Chest. https://doi.org/10.1016/j. chest.2020.04.003

23. Luo L, Luo Z, Jia Y, Zhou C, He J, Lyu J, Shen X (2020) CT differential diagnosis of COVID-19 and non-COVID-19 in symptomatic suspects: a practical scoring method. BMC Pulm Med 20(1):129. https://doi.org/10.1186/s12890-020-1170-6

24. Zhou Z, Guo D, Li C, Fang Z, Chen L, Yang R, Li X, Zeng W (2020) Coronavirus disease 2019: initial chest CT findings. Eur Radiol. https://doi.org/10.1007/s00330-020-06816-7

25. Lomoro P, Verde F, Zerboni F, Simonetti I, Borghi C, Fachinetti C, Natalizi A, Martegani A (2020) COVID-19 pneumonia manifestations at the admission on chest ultrasound, radiographs, and CT: single-center study and comprehensive radiologic literature review. Eur J Radiol Open 7:100231. https://doi.org/10.1016/j.ejro.2020.10023 1

Publisher's Note Springer Nature remains neutral with regard to jurisdictional claims in published maps and institutional affiliations. 